\title{
In the age of electrons, do we still need powder diffraction?
}

\author{
S. Smeets \\ Netherlands eScience Center, Amsterdam, The Netherlands \\ s.smeets@esciencecenter.nl
}

Unit cell determination, phase identification, structure determination, structure refinement. At one point of time, X-ray powder diffraction (XRPD) was the way to go for structure characterization of microcrystalline powders, despite the analyses sometimes being slow and tedious. For a long time, we have known that electron diffraction (ED) data from microcrystals are useful for unit cell and structure determination. We would still resolve to XRPD for structure refinement, because the data are kinematical and therefore simpler to model.

Over the last 15 years, developments in ED methodology, both hardware and software, have reached a point where high-quality data can be collected routinely on a large number of crystals [1,2]. When of sufficient quality, structures refined against these data challenge the accuracy of what can be obtained from XRPD data. By combining data from different crystals using cluster analyses, we showed that even physically meaningful anisotropic ADPs can be obtained from ED data [3]. These are notoriously difficult to obtain from XRPD data.

What can we not do with ED? Through serial crystallography experiments, we saw that it is possible to collect ED data from hundreds or thousands of crystals automatically [2]. This opens the door for automated quantitative phase analysis using ED data [3, 4, 5], challenging the bulk information that can be obtained from XRPD data. Then what do we still need XRPD data for?.

[1] M.O. Cichocka, J. Ångström, B. Wang, X. Zou, S. Smeets, J. Appl. Cryst. 51(6), 1652-1661

[2] B. Wang, X. Zou, S. Smeets, IUCrJ 6(5), 854-867

[3] S. Smeets, S. I. Zones, D. Xie, L. Palatinus, J. C. Pascual, S.-J. Hwang, J. E. Schmidt, L. B. McCusker, Angew. Chem. Int. Ed. 58(37), 1308013086

[4] S. Smeets, J. Ångström, C. O. A. Olsson, Steel Res. Int. 90(1), 1800300

[5] Y. Luo, B. Wang, S. Smeets, J. Sun, W. Yang, and X. Zou, Manuscript in preparation.

Keywords: electron diffraction; powder diffraction; structure refinement; phase analysis 